\title{
FERMAT'S TREATISE ON QUADRATURE: A NEW READING.
}

\author{
JAUME PARADÍS, JOSEP PLA, AND PELEGRÍ VIADER
}

\begin{abstract}
The Treatise on Quadrature of Fermat (c. 1659), besides containing the first known proof of the computation of the area under a higher parabola, $\int x^{+m / n} d x$, or under a higher hyperbola, $\int x^{-m / n} d x$ - with the appropriate limits of integration in each case - has a second part which was not understood by FERMAT's contemporaries. This second part of the Treatise is obscure and difficult to read and even the great HuYGENs described it as "published with many mistakes and it is so obscure (with proofs redolent of error) that I have been unable to make any sense of it". Far from the confusion that HuYGENS attributes to it, in this paper we try to prove that FERMAT, in writing the Treatise, had a very clear goal in mind and he managed to attain it by means of a simple and original method. FERMAT reduced the quadrature of a great number of algebraic curves to the quadrature of known curves: the higher parabolas and hyperbolas of the first part of the paper. Others, he reduced to the quadrature of the circle. We shall see how the clever use of two procedures, quite novel at the time: the change of variables and a particular case of the formula of integration by parts, provide FERMAT with the necessary tools to square very easily curves as well-known as the folium of DESCARTES, the cissoid of DiOcles or the witch of AGNESI.
\end{abstract}

Keywords: History of mathematics. Quadratures. Integration methods Journal of Economic Literature Classification: C00.

MSC 2001 subject classifications. 01A45, 26-03, 26B15, 51M25.

\section{INTRODUCTION}

One of the last papers of FERMAT is devoted to the quadrature of a wide family of algebraic curves, among which the best known and more widely treated by historians are the "higher parabolas and hyperbolas". A curve with equation $y=x^{m / n}$, with $m / n>0$ is what we call a "higher parabola"; if $m / n<0$, we call it a "higher hyperbola". FERMAT's

Date: February 2004.

This paper was written with the support of a grant from the Institut d'Estudis Catalans of the Generalitat de Catalunya, the autonomous government of Catalonia. 
paper also contains an extremely interesting second part concerning the reduction of the quadrature of some curves to the quadrature of others. The paper was written around 1659 and has quite a lengthy title:

De aequationum localium transmutatione et emendatione ad multimodam curvilineorum inter se vel cum rectilineis comparationem, cui annectitur proportionis geometricae in quadrandis infinitis parabolis et hyperbolis usus, (11).

In English,

On the transformation and alteration of local equations for the purpose of variously comparing curvilinear figures among themselves or to rectilinear figures, to which is attached the use of geometric proportions in squaring an infinite number of parabolas and hyperbolas, (translation by Mahoney, (15, p. 245)).

This long title, understandably enough, has been abridged to Treatise on Quadratures (ibid).

It was published in 1679 as part of the complete works of FERMAT collected by his son, Clément-Samuel, (10, pp. 44-57).

FERMAT had done some work on quadratures before. He had tried, unsuccessfully, to square the cycloid and he seems to have already worked on the problem of quadratures in the 1640's as some correspondence with CAvalieri and Torricelli proves (see (15, p. 244)).

The Treatise has been studied before, mainly its first part where, as we have already mentioned, FERMAT presents the integration of $x^{ \pm n / m}$. The second part has been studied in depth in two works: Zeuthen's (24) and Mahoney's (15). Zeuthen's work is a very long paper-in point of fact it is a series of four papers - on different aspects of the history of mathematics. Mahoney's is a book published originally in 1973 with a second printing in 1994. This last work can be considered as the current obliged reference on FERMAT's mathematical work.

Respect the first part of the Treatise we have nothing new to say. It has been thoroughly studied for its great importance within the history of integration since it goes apace with the of other mathematicians of the XVIIth century as Pascal, Cavalieri, Torricelli, Wallis, BARROW, etc. who were working on the problem of the integration of $x^{n}$.

In 1644, according to (24, pp. 41-45), FERMAT was already in possession of the proof of the computation of the quadrature on $[0, b]$ of the parabolas with equation $b^{m} y^{n}=b^{n} x^{m}$, with $m, n$ positive integers, and $b$ a given constant ${ }^{1}$. This was precisely the year that FERMAT sent his results to CAVAlieri via father MERsenne. The complete

\footnotetext{
${ }^{1}$ FERMAT multiplies each side of the equation by the constant $b$ raised to the necessary power in order to maintain the homogeneity of dimensions. See later note 5.
} 
transcription of his work on quadrature into the Treatise must have taken place after 1657, most likely in 1659 (see (15, pp. 244-245, 421) or $(24$, p. 45)), and in that same year he included the quadrature on $[b, \infty)$ of the higher hyperbolas $x^{m} y^{n}=b^{n+m}, m>n$, using an appropriate partitioning of the coordinate axes with the help of geometrical progressions. The details can be found in (15, pp. 245-254), or in (6).

The second part of the Treatise has a much greater interest. Using Mahoney's words:

In this second part, FERMAT grouped together all his mathematical forces - his analytic geometry, his method of maxima and minima, his method of tangents, and his direct quadrature of the higher parabolas and hyperbolasto construct a brilliant "reduction analysis" for the quadrature of curves. $((15$, p. 254$))$

This reflects the importance of this second part where FERMAT develops a true "method" in order to reduce the quadrature of a wide class of algebraic curves to known quadratures among the higher parabolas and hyperbolas as well as the reduction of other curves to the quadrature of a circle. These procedures constitute one of the most interesting lines of research of FERMAT's and their success can be attested by the quadrature of some well-known curves: the folium of DESCARTES, the witch of Agnesi (the versiera or versaria), and the cissoid of Diocles. ${ }^{2}$

Both Zeuthen and Mahoney coincide in pointing out that the words transmutation and alteration in the title of the Treatise clearly show two things:

1) that the Treatise's goal was much more ambitious than the simple quadrature of parabolas and hyperbolas, and

2) that FERmat was imitating VièTE who in his On the Emendation of Equations had studied the solubility of algebraic equations with the help of their transmutation and alteration. FERMAT wanted to do the same with the algebraic equations of curves to determine their "quadrability".

A first reading of the second part of the Treatise leaves one with the impression that FERMAT treats the quadrature of a few particular curves in a disconnected and confused way. Hence, the hasty reader tends to disregard this part of the paper as a simple speculation without real importance. This is exactly what happened to HuYGENS when he read the Treatise the year it was published, 1679. In a letter to Leibniz quoted in $(15$, p. 421)) he says talking of the Treatise:

... this treatise has been published with many mistakes and it is so obscure (with proofs redolent of error) that I have

\footnotetext{
${ }^{2}$ The quadrature of some of these curves is by no means trivial even with our modern integration techniques. See $((17))$.
} 
been unable to make any sense of it. Letter of HUYGENS to

LEIBNiz, 1 September 1691 (21, vol. 4, p. 137).

We shall try to prove that this is not so. Contrarily to these impressions, we hope to show that the whole of the Treatise possesses great depth of thought and the presentation, once understood, shows the great internal coherence of the ideas considered. With FERMAT, we want to emphasize that,

It is remarkable how the theory just presented [the quadrature of the higher parabolas and hyperbolas] can help to advance the work on quadratures since it allows for the easy quadrature of an infinity of curves which no geometer, neither ancient nor modern, has thought of. (11, p. 224).

It is precisely in this second part that we think our article adds something to the existing literature. Despite highlighting the importance of the Treatise, the excellent contributions of Zeuthen (24) and Mahoney (15), cannot devote much space to it since their aim is much broader. Therefore their treatment of the second part of the Treatise is rather descriptive and does not unravel the logical thread that conducts all the examples presented by FERMAT. Zeuthen quite accurately describes the method and each of the individual examples. He neither delves into the method's more delicate aspects nor considers the examples as a whole. Moreover, he does not pay any attention to the question of the limits of integration, which are almost completely disregarded by FERMAT. As far as the method and its details are concerned Mahoney is more thorough, but does not look into more than a couple of examples, missing their coherence as a whole once more.

It is worth mentioning that the Treatise passed unnoticed by FERMAT's contemporaries, ${ }^{3}$ possibly because it was not circulated before its publication. FERMAT probably wrote it in response to WALLIS's Arithmetica Infinitorum of 1656 but there is no mention of it in any of WALLIS's later papers or correspondence, see (15, p. 244).

Without deeper analysis it is difficult to account for the little interest that FERMAT's Treatise aroused in the scientific community of the 1680 's, the time it was published. We dare point out a few, rather obvious reasons why this was so. First, the scientific focus was placed on the calculus of NEWTON and LEIBNIZ, which was flowering with great

\footnotetext{
${ }^{3}$ At the end of his note (3), Aubry remarks that FERMAT's "ingenious procedure of variable substitution as well as his concern to avoid radicals, both in the tracing of tangents and in the quadratures, have had a certain influence on LEIBNIZ and on the updating of his Nova methodus", but he does not substantiate his assertion and we have not been able to find any evidence of it.

Despite the existence of the Treatise and the extant correspondence FERMATCAVAliERI on integration, Andersen in (1) says: "Fermat never disclosed his ideas about the foundation of arithmetical integration". She does not mention any influence between both mathematicians' ideas on integration. We are of the opinion that the question deserves a little more attention.
} 
force at the time. FERMAT's methods, purely algebraic and geometric, based on the comparison of the quadrature of two algebraic curves related by a change of variable, were far from the trend of thought of the new calculus. Second, FERMAT's style is very laconic and, as he was wont to, did not devote much effort or space in making his ideas more comprehensible for the reader. ${ }^{4}$ Even in the cases he solves he does not bother in making the calculations explicit but he limits himself to a mere description of how to attain the desired quadrature. Third, FERMAT's method of quadrature could only be used on a very particular class of curves with a known algebraic equation and did not apply to the more frequent (and trendy) curves of the time: the quadratrix, cycloid, spiral of ARCHIMEDES, etc. Also, FERMAT, contrarily to the rest of authors of the time, did not even refer to infinitesimal quantities, the germ of the new calculus. Finally, the technique of the change of variables, with all certainty, was something new, difficult to grasp and, consequently, suspicious of leading to errors.

In section 2 we undertake the revision of the basis of FERMAT's method which consists of his proof of the linear character of the squaring of sums of parabolas and hyperbolas. Section 3 is devoted to the two instruments of FERMAT's method: a particular instance of the formula of integration by parts and the change of variables. Section 4 tackles the quadrature of the folium of DESCARTES as the first example of the power of the method. This is followed in section 5 by the quadrature of the witch of AGNEsi and the cissoid of Diocles. We reconstruct this last quadrature since FERMAT only mentions in passim that it can be carried out in a similar way to the quadrature of Agnesi's curve. This reconstruction is important because the quadrature of the cissoid reduces to the quadrature of the powers of the ordinates of a circle, that is to say, the integration of functions like $\sqrt[n]{b^{2}-x^{2}}$. This is precisely what is done in section 6 . Section 7 studies the last example presented by FERMAT, a rather involved quadrature that requires several iterations of his method. Lastly, some concluding remarks are offered in section 8 .

\section{FERMAT'S APPROACH}

In the second part of the Treatise, after having learned in the first part how to square higher parabolas and hyperbolas, FERMAT begins by saying that the quadrature of a curve whose equation is the addition or subtraction of different expressions can be squared by the addition or subtraction of the quadrature of each separate summand.

\footnotetext{
${ }^{4}$ This is typical of FERMAT's writings. See (15, p. 25).
} 
Let us consider a curve whose property leads to the following equation $^{5}$

$$
b^{2}-x^{2}=y^{2} .
$$

(It is seen at once that this curve is a circle.)

We can reduce the power of the unknown $y^{2}$ to a root through a division (application ${ }^{6}$ or parabolism). We can indeed write $y^{2}=b u$, as we are free to equate the product of the unknown $u$ and the constant $b$ to the square of the unknown $y$. We will then have

$$
b^{2}-x^{2}=b u \text {. }
$$

But the term $b u$ can decompose in as many terms as those present in the other side of the equation, affecting each one of these terms of the same signs as the corresponding terms of the other side. Let us then write

$$
b u=b i-b \bar{o},
$$

always representing, following Viète, the unknowns by vowels. We will have

$$
b^{2}-x^{2}=b i-b \bar{o} .
$$

Let us equate each one of the terms of one side to the corresponding one in the other side. We will obtain

$$
\begin{aligned}
& b^{2}=b i \text { from which } \quad i=b \quad \text { will be given, } \\
& -x^{2}=-b \bar{o} \quad \text { or } \quad x^{2}=b \bar{o} .
\end{aligned}
$$

The extremity of the line $\bar{o}$ will be on a primary parabola. Thus, in this case, everything can be reduced to a square. If we order all the $y^{2}$ on a given straight line ${ }^{7}$, their sum will be a rectilinear solid, given and known. (11, p. 225)

FERMAT's next example performs the same decomposition to the curve with equation

$$
x^{3}+b x^{2}=y^{3} .
$$

With these two examples, FERMAT has just told us that the sum of all the powers of an ordinate $y^{m}$, when $y^{m}=\sum a_{i} x^{i}$, can be carried out summing each one of the parabolas of the right hand side. The same

\footnotetext{
${ }^{5}$ In this paper the indeterminates $x$ and $y$ will denote the usual rectangular coordinates. Fermat, following Viète, used $E$ and $A$, and Tannery and Henry, in their translation (21) use $e$ and $a$. We prefer to stick to the now traditional $x$ and $y$ in order to make the paper more readable. However we will keep the dimensional homogeneity that FERMAT maintains in all his equations: all the monomials of an algebraic expression must have the same degree in order to be added or subtracted. This is essentially VIÈTE's Homogeneity Law, (23, chap. 3, pp. 2-4). Thus, we will normally use $b$ raised to the necessary powers as a constant that will help us to abide by Viète's law. FERMAT follows Viète very closely on this point but not only as a formal requirement but also, as we will see, as a tool that will help him in his calculations. To know more about the Law of Homogeneity see (12).

${ }^{6}$ On the sense of the term application see later note 9.

${ }^{7}$ The interval on which we sum.
} 
can be made when the right hand side is made of hyperbolas or a sum of parabolas and hyperbolas,

But we obtain not less quadratures by dyeresis $^{8}$, with the help of hyperbolas, either on their own or in combination with parabolas.

He then presents two examples, one that combines parabolas and hyperbolas,

$$
y^{2}=\frac{b^{6}+b^{5} x+x^{6}}{x^{4}}
$$

and the other using only hyperbolas,

$$
y^{3}=\frac{b^{5} x-x^{6}}{x^{3}}
$$

The technique is the same in all cases. If we are interested in calculating the "ordered sum of the $y^{m}$, we "linearize" $y^{m}$ through the term $b^{m-1} u$, that is to say, we effect the change of variable $y^{m}=b^{m-1} u$, and then introduce as many new variables as necessary. For example in the case of the curve with equation (1), the new variables are $\bar{o}, i$ and $\omega$ :

$$
y^{2}=b u=b \bar{o}+b i+b \omega,
$$

which equated term to term with the right-hand side of (1) provide us with three new curves: two hyperbolas,

$$
b^{5}=x^{4} \bar{o}, \quad b^{4}=x^{3} i,
$$

and one parabola

$$
x^{2}=b \omega .
$$

The ordered sum ${ }^{9}$ of the $y^{2}$ of the original curve can be found through the ordered sums of the ordinates of the variables $\bar{o}, i$ and $\omega$ referred to the quadrable two hyperbolas and parabola, i.e. their quadrature. ${ }^{10}$

\footnotetext{
${ }^{8}$ The quadrature by means of parabolas is called syneresis.

${ }^{9}$ We must recall that FERMAT sees the problem of the quadrature of a curve as a purely geometrical problem that can be tackled with the help of algebra. Hence the geometrical language he uses and his geometrical way of thinking. The idea is to sum the ordinates thinking of the area to calculate as the result of putting together all the ordinates that correspond to the curve in question. In that sense, he reminds us of CAVALIERI, with whom he often used to interchange letters, and thus it is not strange that their language resembles. The actual influence of CAVALIERI's method of indivisibles on FERMAT has not been thoroughly studied. Neither Giusti (13) nor Andersen (1, p. 358), mention any evidence of any influence.

One should point out, though, that their summing methods were entirely different. FERMAT, when he speaks of summing ordinates ordered on a given base, understands the sum of an infinity of small rectangles, whereas CAVALIERI's sums of ordinates are a more ambiguous geometrical idea. Mahoney (15, pp. 255-256) translates sum of all the ordinates on a given line as apply all the ordinates to a given line and discusses at length the use of the word "application" in this new context. We refer the interested reader to this work.

${ }^{10}$ It is important to notice that FERMAT makes no comment about the limits of summation of all those expressions. When squaring a parabola, he takes as base
} 


\section{The instruments of Fermat's Method}

After the result of the previous section, which can be described as the "linearity of the summing operation" (the quadrature of a sum is the sum of the quadratures), FERMAT turns to the first essential element in his method of quadratures. We will call it the General Theorem:

Let $A B D N$ be any curve (see Figure 1) with base $H N$ and diameter $H A$. Let $C B, F D$ be the ordinates on the diameter and $B G, D E$ the ordinates on the base.

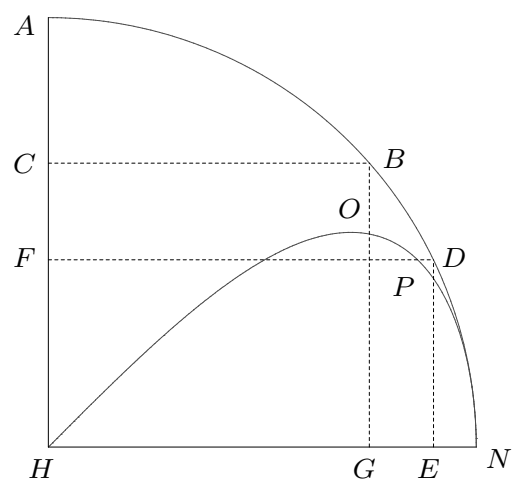

Figure 1.

Let us assume that the ordinates decrease constantly from the base to the summit, as shown in Figure 1; that is to say $H N>F D ; F D>C B$ and so on.

The figure formed by the squares of $H N, F D, C B$, ordered on the line $A H$, that is to say, the solid

$$
C B^{2} \times C A+\cdots+F D^{2} \times F C+\cdots+N H^{2} \times H F+\cdots
$$

is always equal to the figure formed by the rectangles $B G \times$ $G H, D E \times E H$, doubled and ordered on the base ${ }^{11} H N$, that is to say, the solid

$$
2 B G \cdot G H \cdot G H+\cdots+2 D E \cdot E H \cdot E H+\cdots
$$

assuming both series of terms unlimited. As for the other powers of the ordinates, the reduction of the terms on the

the interval $[0, b]$ and when squaring a hyperbola he takes as base the interval $[b, \infty]$ in order to compute the area between the hyperbola and its asymptote (the axis). In this sense, the example we have presented is a little confusing as the presence of both curves in the same quadrature will certainly present problems with the limits of summation. FERMAT completely ignores this objection but later, when he applies the method, he never mixes parabolas and hyperbolas.

${ }^{11}$ FERMAT uses the expression "on the base" or "on the diameter" to indicate, first, the axis on which the infinite partition has to be considered, that is, the modern $d x$ and $d y$; second, it is his reference to the interval on which to carry the summation. When he says, "on the line $b$ " he means the summation on the interval $[0, b]$. 
diameter to the terms on the base is carried out with the same ease; and this observation leads to the quadrature of an infinity of curves unknown till today.

FERMAT's result, in modern notation would amount to

$$
\int_{0}^{b} x^{2} d y=2 \int_{0}^{d} x y d x
$$

He states the result also for the case of the sum of the cubes, $x^{3}$, and the bi-squares, $x^{4}$

$$
\int_{0}^{b} x^{3} d y=3 \int_{0}^{d} x^{2} y d x ; \quad \int_{0}^{b} x^{4} d y=4 \int_{0}^{d} x^{3} y d x .
$$

As the reader can see at once, the General Theorem consists of a geometrical result equivalent in modern language to the following equation, nothing else than a particular case of the formula of integration by parts (we regain the usual role of $x$ and $y$ ):

$$
\int_{0}^{d} y^{n} d x=n \int_{0}^{b} y^{n-1} x d y
$$

where $y(x)$ represents any curve decreasing from the value $b$ to the value 0 as shown in Figure 2. ${ }^{12}$ FERMAT, without stating it, will use the theorem even if the value 0 is reached at infinity, as is the case of Figure 3 for which

$$
\int_{0}^{\infty} x^{n} d y=n \int_{0}^{d} x^{n-1} y d x
$$

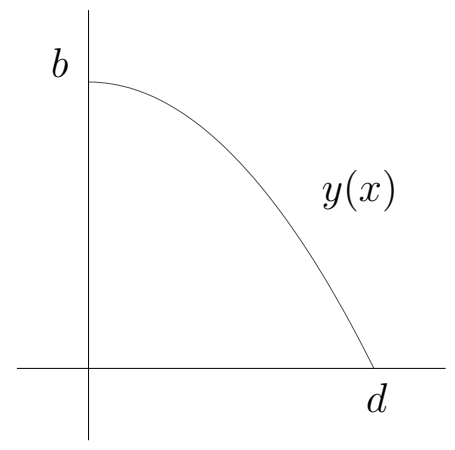

Figure 2.

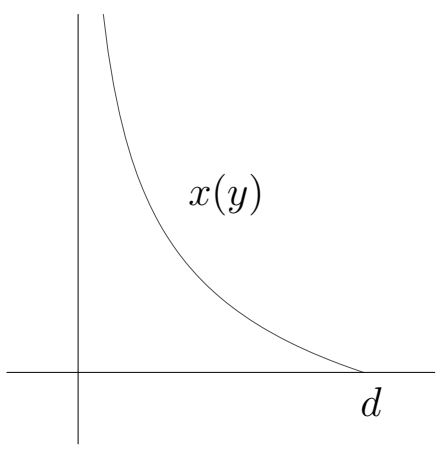

Figure 3.

FERMAT's General Theorem is stated without proof. As can be judged from the quotation, in the case $n=2$ a sort of three-dimensional

\footnotetext{
${ }^{12}$ We will use the modern integral notation to indicate FERMAT's ordered sums. Thus, the sum of $y^{2}$ on the base $x$ will be denoted by $\int y^{2} d x$. We are conscious of the dangers of misinterpretation that this notation has, but the advantages it offers surpass this inconvenience.
} 
argument is used which gives a hint for a possible proof, see (24, p. 51). The cases $n=3$ and $n=4$ are merely worded without more ado.

A geometrical proof of the theorem can be found in a work by PASCAL published in 1659, Traité des trilignes rectangles et des leurs onglets (18, pp. 142-143) or (20, 241-244). PASCAL's result is more general but the proof he offers is consistent with FERMAT's geometrical arguments. It is likely, then, that FERMAT was familiar with PASCAL's theorem and its proof through some correspondence exchanged in 1659 (21, Fermat to Carcavi, 16 February 1659).

With the General Theorem, FERMAT is already in possession of one the keys to his method. In his own words: "From here, as we will see, there will derive an infinite number of quadratures".

The second instrument he needs is the transformation of equations ${ }^{13}$ with help from the technique of the change of variables.

The first example he offers begins with the equation of a circle.

Let, for instance, $b^{2}-y^{2}=x^{2}$ be the equation that constitutes the curve (which will be a circle). According to the general theorem above, the sum of the $x^{2}$ ordered on the line $b$ [the diameter] equals the sum of the products $H G \cdot G B$ [Figure 1] doubled and ordered on the line $H N$ or $d$ [the base]; but the sum of the $x^{2}$, ordered on $b$ equals, as has been proven above, a given rectilinear area. Consequently, the sum of the products $H G \cdot G B$, doubled and ordered on the base $d$ constitute a given rectilinear area. If we half it, the sum of the products $H G \cdot G B$, ordered on the base $d$ will also constitute a given rectilinear area. (11, pp. 228-229)

FERMAT applies his General Theorem (to $x(y))$ and obtains the result (in the case of his example, $b^{2}-y^{2}=x^{2}$ )

$$
\int_{0}^{d} x y d x=\frac{1}{2} \int_{0}^{b} x^{2} d y .
$$

In this particular case $d=b .{ }^{14}$ But none of these two "summations" corresponds, from FERMAT's point of view, to a proper ordered sum of ordinates applied to a segment. For this reason he needs to "linearize" the product $x y$ in order to have a properly quadrable (and new) curve.

This is the purpose of the second essential element of his method, the change of variables.

In order to pass easily and without the burden of radicals ${ }^{15}$

from the first curve to the new one, we have to employ an

\footnotetext{
${ }^{13}$ Notice that the main emphasis in the title of the Treatise is on the transformation and alteration of equations. See the introduction.

${ }^{14}$ We must keep in mind that FERMAT always uses examples to present theoretical results. Thus, while the example he offers refers to the circle, he speaks as if the curve were the curve $A B D N$ of the General Theorem.

${ }^{15}$ This is one of the important consequences of FERMAT's method: the sum of radical powers of ordinates avoiding the use of radicals. In this first example,
} 
artifice which is always the same and which is the essence of our method.

Let $H E \cdot E D$ [Figure 1] be any of the products we have to order on the base. In the same way that we call analytically $x$ the ordinate $F D$ or its parallel $H E$ and we call $y$ the coordinate $F H$ or its parallel $D E$, we will call $x y$ the product $H E \cdot E D$. Let us equate this product $x y$, formed by two lines unknown and undetermined, to $b u$, that is to say, the product of the given $b$ by an unknown $u$ and let us suppose that $u$ equals $E P$ taken on the same line that $D E$. We will have

$$
\frac{b u}{x}=y
$$

But according to the specific property of the first curve, ${ }^{16}$ $b^{2}-y^{2}=x^{2}$. Replacing $y$ by its new value $b u / x$ we will have $b^{2} x^{2}-b^{2} u^{2}=x^{4}$ or, transposing,

$$
b^{2} x^{2}-x^{4}=b^{2} u^{2},{ }^{17}
$$

equation that constitutes the new curve $H O P N$ [Figure 1], derived from the first. For this curve it is proved that the sum of the $b u$ ordered on $b$ is given. Dividing by $b$, the sum of the $u$ ordered on the base, that is to say, the surface $H O P N$ will be given as a rectilinear area and we will consequently obtain its quadrature.

FERMAT effects the change of variable ${ }^{18} y=b u / x$ and the circle transforms into a new curve in the $x u$-plane (see Figure 4 ).

FERMAT wants to calculate

$$
\int_{0}^{b} x \sqrt{b^{2}-x^{2}} d x
$$

The change of variable will "linearize" $x y$ and convert it to $b u$, where $u$ will be the ordinate of a new curve. See (24, p. 55).

${ }^{16}$ That is to say, its analytic equation.

${ }^{17}$ Notice that $b u=x \sqrt{b^{2}-x^{2}}$.

${ }^{18}$ Notice the homogeneity of the dimensions. The constant $b$ is introduced not only to keep the dimensions right but also to keep the "limit" of summation under control. Notice that the point $(b, 0)$ in the $x y$-plane becomes $(b, 0)$ in the $x u$-plane. 


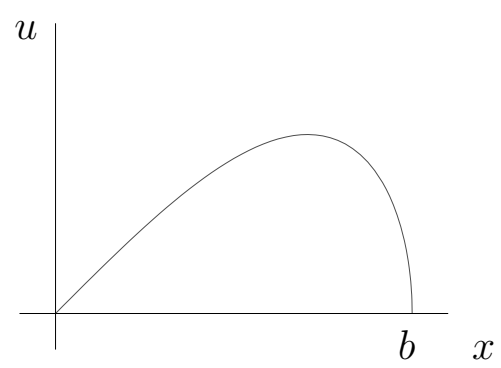

Figure 4. The new curve, $b^{2} x^{2}-x^{4}=b^{2} u^{2}$.

As the change amounts to $x y=b u$, the new curve is quadrable for the new ordinates $u$ can be summed when ordered on the line $b$ :

$$
\int_{0}^{b} u d x=\frac{1}{b} \int_{0}^{b} x y d x=\frac{1}{2 b} \int_{0}^{b} x^{2} d y,
$$

and as the sum of the $x^{2}$ can be obtained squaring two parabolas,

$$
\int_{0}^{b} x^{2} d y=\int_{0}^{b}\left(b^{2}-y^{2}\right) d y=\frac{2 b^{3}}{3}
$$

we have

$$
\int_{0}^{b} u d x=\frac{b^{2}}{3}
$$

FERMAT's method in this first example consists essentially of the following. We start from an algebraic equation $y^{n}=\sum a_{i} x^{i}+\sum b_{j} / x^{j}$ and, consequently we know how to calculate $\int_{0}^{b} y^{n} d x$. We then proceed in two steps:

1) Apply the General Theorem: $\int_{0}^{b} y^{n} d x=n \int_{0}^{d} y^{n-1} x d y$;

2) Linearize the integrand through an appropriate change of variable: $y^{n-1} x=b^{n-1} u$, where $u$ is the ordinate of a new - and $a$ fortiori, quadrable - curve.

In FERMAT's account it is worth mentioning the absolute lack of references to the region which is actually squared in each curve. It goes without saying that if the curve draws a closed region this is precisely the area to be squared. If the curve has an asymptote, the region to be squared is the one trapped by the curve, the asymptote (which is always an axis) and an appropriate ordinate which is almost self-evident. ${ }^{19}$

The next example presented by FERMAT follows the same pattern: an application of the General Theorem and a change of variables. The starting curve is the cubic

$$
y^{3}=b x^{2}-x^{3}
$$

\footnotetext{
${ }^{19}$ FERMAT only considers positive values of the variables and consequently, his quadratures limit themselves to the first quadrant.
} 
The new curve, however, ends up being an algebraic curve of degree 9 in the variable $y$ and degree 3 in the new variable $u$. The squaring of this last curve is a challenge even if one is equipped with all the artillery our calculus provides us with.

FERMAT offers this example not only as a second instance of his method but also to exemplify a situation which needs an improved version of the General Theorem.

FERMAT reminds us that the sum of all $y^{3}$ on the interval $[0, b]$ is immediately obtained as a sum of two quadrable parabolas (see Figure $5)$,

$$
\int_{0}^{b} y^{3} d x=\int_{0}^{b}\left(b x^{2}-x^{3}\right) d x=\frac{b^{4}}{12}
$$

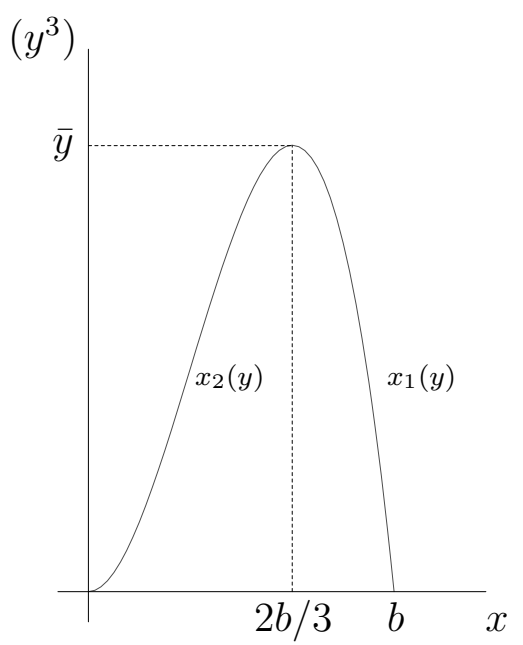

Figure 5. $f(x)=b x^{2}-x^{3}$.

On the other hand, the General Theorem says that

$$
\int_{0}^{b} y^{3} d x=3 \int_{0}^{\bar{y}} y^{2} x d y .
$$

FERMAT now makes the change of variable that provides the linearization part of the method,

$$
x=\frac{b^{2} u}{y^{2}}
$$

which takes the curve (5) into the curve with equation (see Figure 6):

$$
b^{5} u^{2} y^{2}-y^{9}=b^{6} u^{3} .
$$




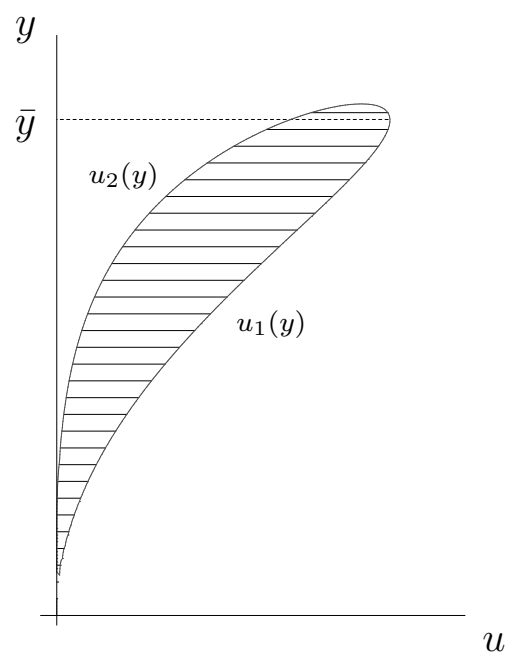

Figure 6. $b^{5} u^{2} y^{2}-y^{9}=b^{6} u^{3}$.

The change effected on the integral of the right-hand side of (6) is

$$
\int_{0}^{b} y^{2} x d y=b^{2} \int_{0}^{\bar{y}} u d y
$$

Notice the upper limit of integration in the new integral: $\bar{y}$. If you look at Figure 6, you will clearly see that the "sum of all the $u$ " has to be "ordered on the line $\bar{y}$ ". But actually, the value of $\bar{y}$ is irrelevant for FERMAT's purposes as the quadrature he is interested in is represented exactly by that last integral which will be calculated going backwards in the chain of integrals obtained so far:

$$
\int_{0}^{\bar{y}} u d y=\frac{1}{b^{2}} \int_{0}^{\bar{y}} y^{2} x d y=\frac{1}{3 b^{2}} \int_{0}^{b} y^{3} d x=\frac{b^{2}}{36}
$$

Thus, the quadrature of the new curve is $b^{2} / 36$.

As we mentioned before, in this example, some comments are really necessary to fully understand FERMAT's technique.

The initial curve, $y^{3}=b x^{2}-x^{3}$, is not decreasing, a necessary condition for the General Theorem to hold. In fact, seen as a function $y(x)$ it increases on the interval $[0,2 b / 3]$, and decreases from there until reaching the value 0 for $x=b$. The highest value it attains is $\bar{y}=\sqrt[3]{4 b^{2}} / 3$. In terms of $y^{3}$, this maximum is, obviously, $\bar{y}^{3}=4 b^{2} / 27$.

This means that when $y$ varies between 0 and $\bar{y}$, for each value of the variable $y$, two values are obtained for the variable $x$. Let us denote each of these values by $x_{1}$ and $x_{2}$, as shown in Figure 5 . We can think of $x_{1}(y)$ and $x_{2}(y)$ as two different functions. The same considerations have to be made about the new curve, see Figure 6. Again, for a given $y$, two values of $u$ have to be considered, $u_{1}$ and $u_{2}$. 
Thus, to be rigorous, FERMAT's procedure should be rewritten as follows:

$$
\int_{0}^{b} y^{3} d x=3 \int_{0}^{\bar{y}} y^{2}\left(x_{1}-x_{2}\right) d y=3 b^{2} \int_{0}^{\bar{y}}\left(u_{1}-u_{2}\right) d y
$$

this last integral representing the lined area in Figure 6.

FERMAT is conscious that this example is not exactly covered in his General Theorem and proceeds to offer a new version when the curve is not decreasing. His explanation amounts to saying that if a curve as the one shown in Figure 7a) is given, the General Theorem can be applied first to the decreasing portion of the curve, $x_{2}(y)$ from $x=0$ to $x=z$ where the maximum is reached. A different procedure, though, has to be used for the increasing portion, the one we have called $x_{1}(y)$ that increases from $x=b$ to $x=z$. Essentially what FERMAT does is change the axes in such way as to have the increasing portion as a decreasing curve. Consider $x=z$ as the new "base". On the one hand we have the curve $z-x_{2}$ which is decreasing from the new base to $x=z$ (we have to think of positive $x$ downwards), see Figure 7b; and on the other hand we have the curve $x_{1}-z$ which decreases from the new base to $x=b-z$, see Figure $7 \mathrm{c}$. The sum of the $y^{n}$ ordered on $[0, b]$ can obviously be decomposed into the two portions.

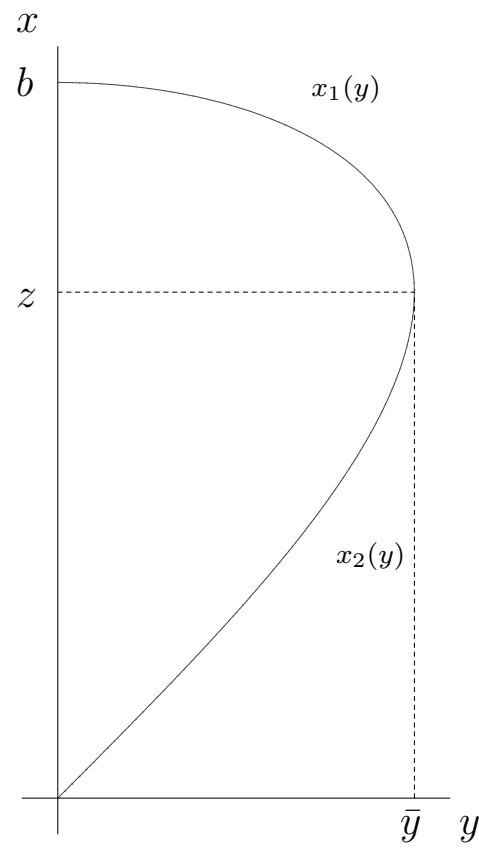

Figure 7a.

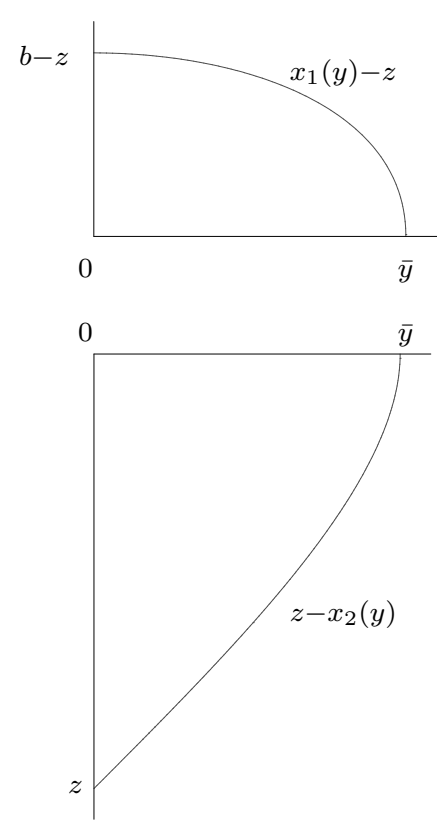

Figure $7 \mathrm{~b}$ and $7 \mathrm{c}$. 
His arguments, translated into our notation would lead to:

$$
\begin{aligned}
\int_{0}^{b} & y^{n} d x=\int_{0}^{z} y^{n} d x+\int_{z}^{b} y^{n} d x \\
\quad= & n \int_{0}^{\bar{y}} y^{n-1}\left(x_{1}-z\right) d y+n \int_{0}^{\bar{y}} y^{n-1}\left(z-x_{2}\right) d y \\
\quad & n \int_{0}^{\bar{y}} y^{n-1}\left(x_{1}-x_{2}\right) d y .
\end{aligned}
$$

Obviously, the value $z$ of $y$ where the maximum $\bar{y}$ for $x$ is attained can be obtained by his method of maxima and minima, developed twenty years before. Paradoxically, these values are of no importance as they only are intermediate values that are not explicitly needed to carry out the quadrature ${ }^{20}$. This is probably one of the reasons why FERMAT pays no attention at all to the limits of summation in the intermediate curves he uses.

\section{The QuAdrature of The Folium of Descartes.}

The next example FERMAT offers has the clear intention of creating an impression on the reader.

Just to clearly show that our method provides new quadratures, which had never even been suspected before among the moderns, let the curve before considered be proposed ${ }^{21}$ with equation

$$
\frac{b^{5} x-b^{6}}{x^{3}}=y^{3} .
$$

It has been proved that the sum of the $y^{3}$ is given as a rectilinear area. Transforming them on the base ${ }^{22}$ we will have, according to the preceding method, $b^{2} u / y^{2}=x$. Replacing the new value of $x$ and finishing the calculations according to the rules, ${ }^{23}$ we will arrive at the new equation $y^{3}+u^{3}=b y u$, which provides a curve from the side of the base. It is the one from Schooten, who gave its construction in his Miscellanea, section XXv, page $493 .{ }^{24}$ The curvilinear figure $A K O G D C H$ of this author is, consequently, easily quadrable according to the preceding rules.

\footnotetext{
${ }^{20}$ Mahoney (15, p. 264) says on this point that "Fermat employs his method of maxima and minima to determine the value of $x$ for which $y$ attains a maximum and the value of that maximum." This is not really so as the actual values of both, the maximum and the value of $x$ where it is attained, are irrelevant in FERMAT's method.

${ }^{21}$ It is example (2).

${ }^{22}$ That is, using the General Theorem.

${ }^{23}$ The rules of algebra, of course.

${ }^{24}$ The curve is the folium of Descartes. We coincide with (15, p. 265, n. 67) when he insinuates that FERMAT deliberately slights DESCARTES as the author of the curve and attributes it to van ScHOOTEN.
} 
The folium of DESCARTES appeared during the controversy that confronted FERMAT and DESCARTES around 1637 on the methods for the tracing of tangents to curves. After quite an acrid exchange of letters with examples and counterexamples to prove the superiority of each other's method, DescarTes ended by challenging FERMAT to find the tangents to the curve of his invention with equation (see Figure 8)

$$
x^{3}+y^{3}=b x y .
$$

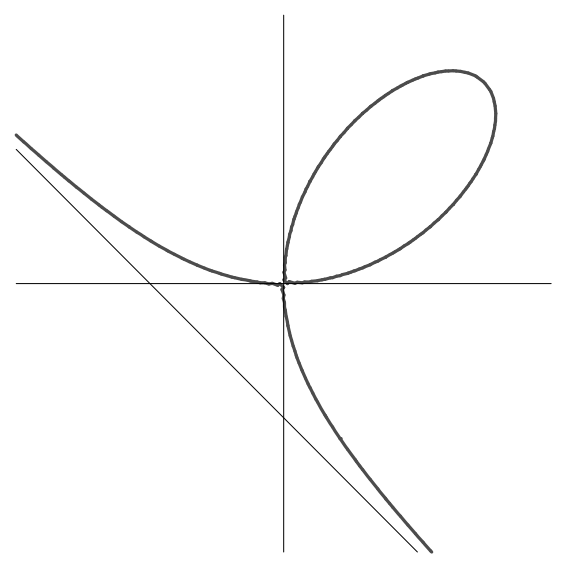

Figure 8. The folium of Descartes, $x^{3}+y^{3}=b x y$.

FERMAT not only solved the problem but offered a general solution that allowed him to find the two tangents of a given slope (see (8) or (15, p. 181 and ff) for more details about the controversy). DESCARTES, after this tour de force of his opponent, had to admit FERMAT's superiority and the merit of being one of the greatest geometers of the moment. It is not strange, then, that twenty years later, FERMAT used the same curve to prove once more the mettle of his genius.

If you follow the exasperatingly short description of FERMAT in the quotation above, one realizes that FERMAT starts with an apparently innocent curve that, as if by chance, gets transformed into the equation of the folium. It is obvious that FERMAT proceeded just in the contrary direction. From the equation of the folium, he derived an equation which had the necessary features for his method to be applied, that is, an equation of the form

$$
y^{m}=\sum a_{i} x^{i}+\sum \frac{b_{j}}{x^{j}} .
$$

In the case of the folium, FERMAT's most likely train of thought would have been to essay a change of variable that replaced $x$ in (7) by an expression involving the new variable $u$ and the old $y$ in such a way that after making the change the new equation would look like (8). In 
order to achieve this it is enough to make the change of variable ${ }^{25}$

$$
x=\frac{u y^{2}}{b^{2}}
$$

which alters (7) into

$$
\frac{u^{3} y^{6}}{b^{6}}+y^{3}=\frac{u y^{3}}{b}
$$

or, after simplifying $y^{3}$ from each side and rearranging,

$$
y^{3}=\frac{b^{5}(u-b)}{u^{3}} .
$$

The graph of $y^{3}$ as a function of $u$ can be seen in Figure $9 \mathrm{~b} .{ }^{26}$

We can now present the chain of integrals of FERMAT's method (see Figures $9 \mathrm{a}$ and 9b):

$$
\begin{aligned}
\int_{0}^{\bar{y}}\left(x_{1}-x_{2}\right) d y & =\frac{1}{b^{2}} \int_{0}^{\bar{y}}\left(u_{1}-u_{2}\right) y^{2} d y \\
& =\frac{1}{3 b^{2}} \int_{b}^{\infty} y^{3} d u=\frac{b^{3}}{3} \int_{b}^{\infty} \frac{u-b}{u^{3}} d u=\frac{b^{2}}{6} .
\end{aligned}
$$

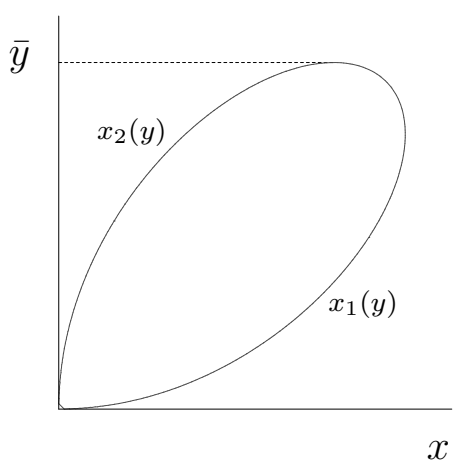

Figure 9a. $x^{3}+y^{3}=b x y$.

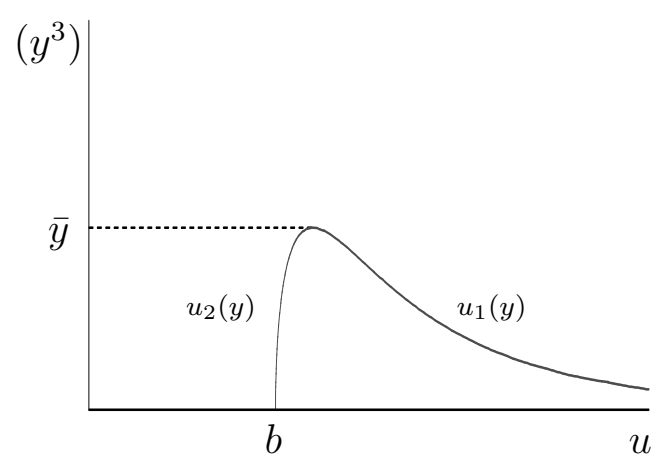

Figure $9 \mathrm{~b} . f(u)=b^{5}(u-b) / u^{3}$.

We can also ask ourselves (see (17)) about the possibility that FERMAT carried out a few trials on curves composed by higher hyperbolas with equations of the form:

$$
y^{m}=\frac{b^{m+k-1}(x-b)}{x^{k}} ; \quad m \geq 2, \quad k>2 .
$$

\footnotetext{
${ }^{25}$ The change $x=b^{2} u / y^{2}$ is an alternative that also solves the problem. Bernoulli, in his (4, p. 403) uses this last change of variable in order to square the folium, but here ends all similitude with FERMAT's method, despite what Aubry says in (3). See also footnote 30 .

${ }^{26}$ It must be noticed that equation (9) has a very special structure, which, as we will see, occurs almost in the same form in many of FERMAT's examples.
} 


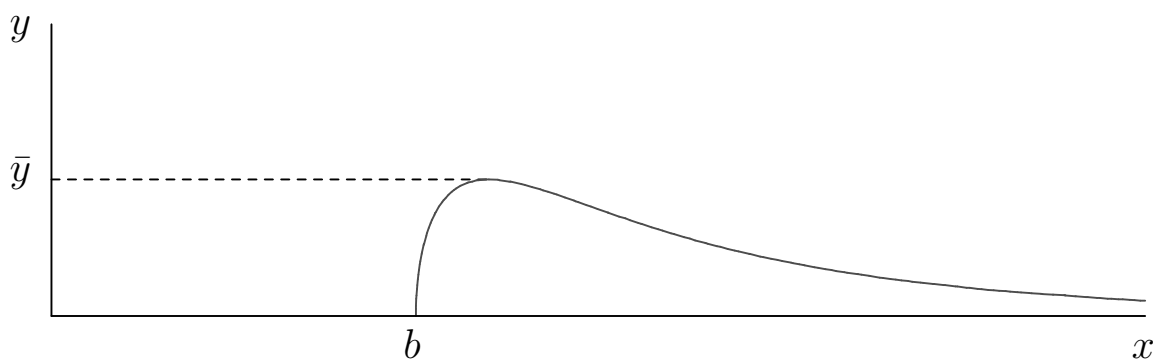

Figure 10.

The graph in Figure 10 corresponds to $y$ in (10). The graph of $y^{m}$, for $m>0$, is essentially the same.

Proceeding à la FERMAT we make the change of variable

$$
x=\frac{b^{m-1} z}{y^{m-1}}
$$

and we undertake the chain of integrals:

$$
\int_{b}^{\infty} y^{m} d x=m \int_{0}^{\bar{y}} y^{m-1} x d y=m b^{m-1} \int_{0}^{\bar{y}} z d y .
$$

The new curve's equation will be

$$
b^{(m-2) k-m} z^{k}+y^{(m-1) k-m}=b^{m-2} z y^{(m-1)(k-1)-m} .
$$

This family of curves, in the first quadrant have a loop similar to the loop of the folium - which is the curve given by $m=3$ and $k=3$. The areas of these loops, that is to say

$$
\int_{0}^{\bar{y}} z d y
$$

are

$$
A(m, k, b)=\frac{b^{2}}{m(k-1)(k-2)} .
$$

It is seen at once that FERMAT's method also solves in a quite straightforward way the quadrature of the generalized folia of (7) with equation

$$
x^{2 q+1}+y^{2 q+1}=(2 q+1) b x^{q} y^{q},
$$

where $q$ is a positive integer.

The change of variable required is $b^{q+1} x^{q}=u^{q} y^{q+1}$, and the areas of the loops in the first quadrant are

$$
A(q, b)=\frac{2 q+1}{2} b^{2} .
$$

More details can be found in (17). 


\section{The quadrature of the Witch of Agnesi And the Cissoid of Diocles.}

FERMAT next tackles the quadrature of the curve known today as the witch of AGnesi. ${ }^{27}$ This curve seems to have been brought to FERMAT's attention by the geometer LALOUVÈRE who might have asked FERMAT about its quadrature, ${ }^{28}$ and immediately after that FERMAT says: "With the same method I have squared the cissoid of DiOCLES or, I had rather say that I have reduced its quadrature to that of the circle." 29

FERMAT in the Treatise does not give any more indications about how he reached the quadrature of the cissoid. ${ }^{30}$ Notwithstanding, the two curves, the versiera and the cissoid, have similar cartesian equations, a fact that makes a common treatment with FERMAT's method possible. In fact, we will treat a more general family of curves which can be tackled in the same way.

Let us consider the family of curves $^{31}$ with equation

$$
b^{N-3} x y^{2}=(b-x)^{N} .
$$

For $N=1$ we have the versiera (Figure 11) and for $N=3$ the cissoid (Figure 12).

\footnotetext{
${ }^{27}$ This curve was studied in 1748 by Maria Gaetana AGNESI (1718-1799) and had already been object of attention by Guido Grandi (1703) who gave it the curious name of versiera or versaria $($ see $(16 ; 22 ; 14)$ for the history of the name and other details about the curve itself). In English it is known as the witch of AGNESI or the curve of AGNESI.

${ }^{28}$ FERMAT in the Treatise on quadratures (21, p. 234), after constructing geometrically the versiera and after giving us the value of its quadrature, comments: "It is so that we have solved at once that question proposed to us by a learned geometer". (2, p. 85) is of the opinion that the "learned geometer" mentioned by Fermat is none other than Antoine De Lalouvère, a Jesuit from Toulouse and frequent correspondent of FERMAT. Anyway, we have not been able to find a previous mention of a curve like the versiera in the literature, and this leads us to think that FERMAT might be the real author of the curve, at least of its algebraic equation.

${ }^{29}$ The same FERMAT in (9) squares the cissoid by purely geometrical methods without using any of the methods of the Treatise. The result he obtains in that brief "fragment" comes to say that the area trapped between the cissoid and it asymptote is the triple of the area of the semicircle used in its geometrical construction. Details of this construction can be found in (22).

${ }^{30}$ Aubry (3) offers a reconstruction of the quadrature of the cissoid of doubtful likelihood. Aubry freely uses differentials and the full formula of integration by parts, poles apart from FERMAT's method. More than from FERMat, Aubry seems to borrow from JoHn Bernoulli, who in (4, pp. 399-407) had tackled the quadrature of the folium, the versiera and some other curves treated by FERMAT. BERNOULLI's procedure, though vaguely reminiscent of FERMAT by the changes of variables used, is definitely far from the method of the French mathematician.

${ }^{31}$ Notice that these curves are again of the form (10). The only difference is that instead of $x-b$, now we consider $b-x$.
} 


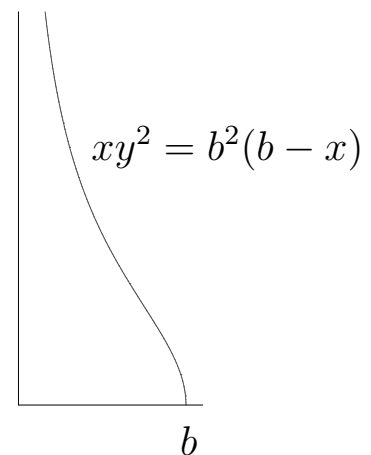

Figure 11. Versiera.

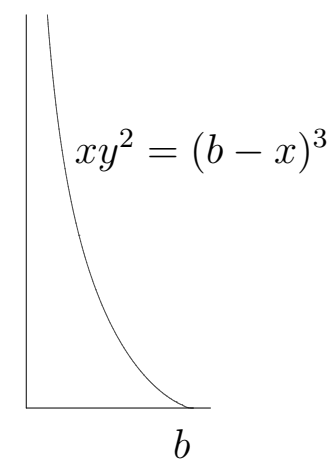

Figure 12. Cissoid.

The quadrature of the family of curves (11) will correspond to the area trapped between the curve and the two axes - the vertical axis is in fact the asymptote of the curve. It can be obtained with the help, in this case, of two changes of variables. We will use a $\mathrm{T}$ on the equal sign to denote an application of the General Theorem and CV to denote the corresponding change of variables.

$$
\begin{aligned}
\text { Area } & =\int_{0}^{\infty} x d y \\
& \stackrel{\mathrm{CV} 1}{=} \frac{1}{b} \int_{0}^{\infty} z^{2} d y \stackrel{\mathrm{T}}{=} \frac{2}{b} \int_{0}^{b} y z d z \stackrel{\mathrm{CV} 2}{=} \frac{2}{b^{N-1}} \int_{0}^{b} u^{N} d z .
\end{aligned}
$$

FERMAT needed two changes of variable:

$$
\text { CV1: } \quad x=\frac{z^{2}}{b}
$$

which led to the new curve

$$
b^{2 N-4} z^{2} y^{2}=\left(b^{2}-z^{2}\right)^{N},
$$

and

$$
\text { CV2: } \quad y=\frac{u^{N}}{b^{N-2} z}
$$

giving the last curve which, independently of $N$, is the same circle,

$$
u^{2}=b^{2}-z^{2} \text {. }
$$

Now, the quadrature of our first curve will depend on the sum of all the $u^{N}$ on the interval $[0, b]$,

$$
\int_{0}^{b} u^{N} d z
$$

where $u$ is the ordinate of a circle of radius $b$. For even values of $N$, it is clear that the required sum will be very easy to calculate as 
it will ultimately be a sum of quadratures of parabolas, the powers $\left(b^{2}-z^{2}\right)^{N / 2}$. For odd values of $N$, the required sum will not be so easy to carry out. ${ }^{32}$

The simplest odd case, the case of the versiera $(N=1)$, is easily dealt with. Its quadrature will depend on the quadrature of the circle itself, (formula (12) for $N=1$ ))

$$
\text { Area versiera }=2 \int_{0}^{b} u d z=\frac{\pi b^{2}}{2} .
$$

For the rest, as the case of the cissoid $(N=3)$ demands, FERMAT will now deal with the problem of summing different powers of the ordinates of a circle. ${ }^{33}$

\section{The sum of the Powers of the ordinates of A CirCle}

FERMAT, apparently, stops analyzing the quadrature of curves and turns to solve the problem of finding the sum of the powers of the ordinate of a circle, which is absolutely coherent with his previous calculation.

He begins by considering the equation of the circle $y^{2}=b^{2}-x^{2}$. He has already remarked that the sum of even powers of $y$ are no problem. The odd powers, he asserts, can be reduced through his method to the quadrature of the circle.

FERMAT considers only the case $y^{3}$ and informs us that the generalization to all odd powers is very easy.

As (24, pp. 57-58) says, FERMAT's method reduces the sum of $y^{2 n+1}$ to the sum of $z^{n}$ where $z$ is the ordinate of a circle of radius $b / 2$ and not centered on the origin. This reduction is faster than the one we would undertake today if we had to calculate

$$
\int_{0}^{b}\left(b^{2}-x^{2}\right)^{(2 n+1) / 2} d x
$$

integrating directly by parts. This would imply the differentiation of $\left(b^{2}-x^{2}\right)^{(2 n+1) / 2}$ and a reduction formula that reduces the degree in 2 units at a time. ${ }^{34}$ FERMAT's reduction, as we will see, halves the degree each time.

\footnotetext{
${ }^{32}$ Here FERMAT again faces the problem of the sum of a radical power of the ordinates. His method circumvents the difficulty.

${ }^{33}$ FERMAT, of course, does not inform us that the sum of the odd powers of the ordinates of the circle is needed to square the cissoid!

${ }^{34}$ If we bother to do the necessary calculations, we get the reduction formula

$$
\int_{0}^{b}\left(b^{2}-x^{2}\right)^{(2 n+1) / 2} d x=\frac{(2 n+1) b^{2}}{2 n+2} \int_{0}^{b}\left(b^{2}-x^{2}\right)^{(2 n-1) / 2} d x .
$$
}


Let $A(m, r)$ denote the sum of the $y^{m}$ where $y$ is the ordinate of a circle of radius $r$ centered on the origin.

$$
\begin{aligned}
& A(2 n+1, b)=\int_{0}^{b} y^{2 n+1} d x \\
& \stackrel{\mathrm{T}}{=}(2 n+1) \int_{0}^{b} y^{2 n} x d y \stackrel{\mathrm{CV} 1}{=}(2 n+1) b \int_{0}^{b} y^{2 n-1} u d y \\
& \stackrel{\mathrm{T}}{=} \frac{2 n+1}{2 n} \int_{0}^{b / 2} y^{2 n} d u \stackrel{\mathrm{CV} 2}{=} \frac{2 n+1}{2 n} b^{n} \int_{0}^{b / 2} v^{n} d u .
\end{aligned}
$$

The changes of variable indicated are:

$$
\text { CV1: } \quad x=\frac{b u}{y}
$$

which leads to the new curve

$$
b^{2} u^{2}=y^{2}\left(b^{2}-y^{2}\right),
$$

and

$$
\text { CV2: } \quad y^{2}=b v
$$

which produces the curve

$$
u^{2}=b v-v^{2} .
$$

Let us remark that this last curve is a circle of center $(b / 2,0)$ and radius $b / 2$. The sum of the $v^{n}$, where $v$ is the ordinate of this circle, has to be taken as the sum of the expressions $v_{1}^{n}-v_{2}^{n}$, where the $v_{i}$ are the monotone portions of the circle as shown in Figure 13.

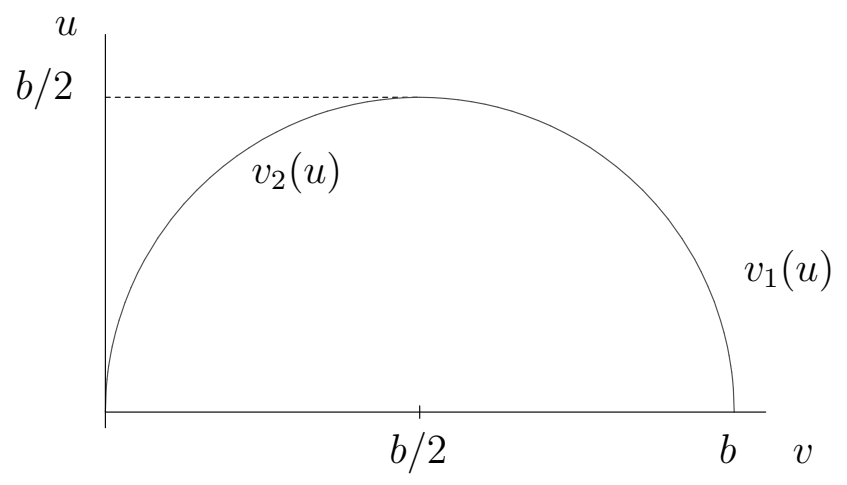

Figure 13. $u^{2}=b v-v^{2}$.

Since FERMAT presents only the case $n=1$, he finds no difficulties as the sum of the $v$ is simply half the area of a circle of radius $b / 2$. But for $n>1$ one must still reduce the new circle to another circle, this time, 
centered on the origin in order to be able to iterate the procedure. This can be done with another change of variable:

$$
\mathrm{CV} 3: \quad v_{1}=\frac{b}{2}+t, \quad v_{2}=\frac{b}{2}-t
$$

which transforms the sum of the $v^{n}$ as follows:

$$
\begin{aligned}
\int_{0}^{b / 2}\left(v_{1}^{n}-v_{2}^{n}\right) d u \stackrel{\mathrm{CV} 3}{=} \int_{0}^{b / 2}\left\{\left(t+\frac{b}{2}\right)^{n}-\left(t-\frac{b}{2}\right)^{n}\right\} d u \\
=2 \sum_{j=1}^{\lceil n / 2\rceil}\left(\begin{array}{c}
n \\
2 j-1
\end{array}\right)\left(\frac{b}{2}\right)^{n-2 j+1} \int_{0}^{b / 2} t^{2 j-1} d u
\end{aligned}
$$

The sum of the odd powers of $t$ correspond to the circle centered on the origin with equation $t^{2}=(b / 2)^{2}-u^{2}$. We obtain a recurrence formula for the sum of the odd powers of the ordinates of a circle:

$$
A(2 n+1, b)=\frac{2 n+1}{n} \sum_{j=1}^{\lceil n / 2\rceil}\left(\begin{array}{c}
n \\
2 j-1
\end{array}\right)\left(\frac{b}{2}\right)^{n-2 j+1} \cdot A(2 j-1, b / 2) .
$$

In this last formula, $\lceil x\rceil$ denotes the ceiling of the number $x$, to wit, the smallest integer greater than or equal to $x$.

\section{THE LAST TURN OF THE SCREW.}

FERMAT, to close his paper yields to the temptation of presenting the quadrature of a curve that needs up to eight changes of variable to be reduced.

As for the rest, it often occurs that, strangely enough, in order to reach the simple measure for a proposed equation of locus we need to carry our analysis through a great number of curves.

This last example is, obviously enough, a tour de force to present an almost impossible quadrature. But after careful analysis we can see that it is not only that. It can be placed along the class of curves that lead to the quadrature of the folium of DESCARTES. The difference lies in the fact that now FERMAT wants to find the quadrature of the first curve instead of starting with the known sum of the power of the ordinates of a curve in order to derive the quadrature of a new curve. In this, the example differs from the previous ones. 
FERMAT's initial equation is ${ }^{35}$

$$
y^{2}=\frac{b^{7}(x-b)}{x^{6}}
$$

The aim of FERMAT is to square this curve (see Figure 14), that is, to compute

$$
\int_{b}^{\infty} y d x
$$

or, what amounts to the same,

$$
\int_{0}^{\bar{y}} x d y
$$

Instead of studying FERMAT's curve directly, we will deal with his example a little more generally. This greater perspective will help us in our analysis.

Let us the consider the curve (see Figure 14) with equation

$$
y^{2}=\frac{b^{k+1}(x-b)}{x^{k}}
$$

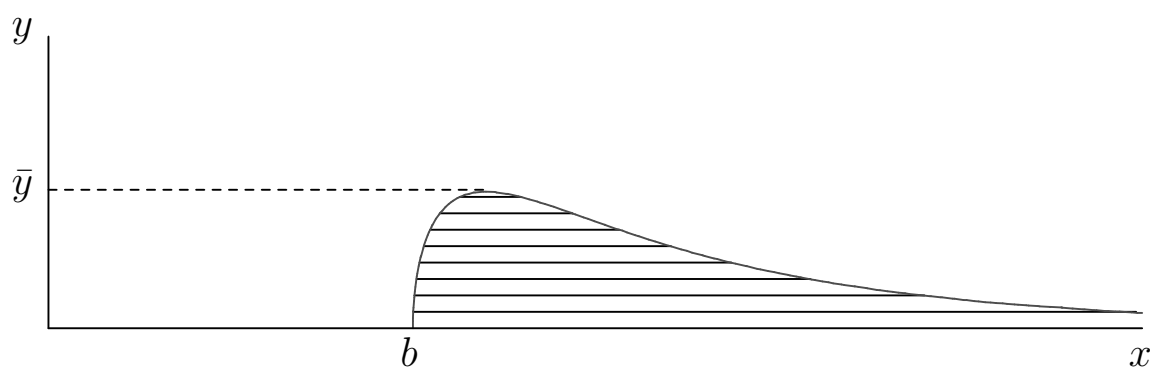

Figure 14. $y^{2}=b^{k+1}(x-b) / x^{k}$.

\footnotetext{
${ }^{35}$ Let us notice that this equation is again a generalization of the curves of the form

$$
y^{m}=\frac{b^{m+k-N}(b-x)^{N}}{x^{k}} .
$$

This remark makes us consider the possibility that FERMAT was conscious of the generality of his method for this type of curves.
} 
The chain of integrals and the corresponding changes of variable with the resulting curves is the following:

$$
\begin{aligned}
& \int_{0}^{\bar{y}} x d y \stackrel{\mathrm{CV} 1}{=} \quad \frac{1}{b} \int_{0}^{\bar{y}} z^{2} d y \stackrel{\mathrm{T}}{=} \frac{2}{b} \int_{b}^{\infty} z y d z \stackrel{\mathrm{CV} 2}{=} \frac{2}{b} \int_{b}^{\infty} u^{2} d z \\
& \stackrel{\mathrm{T}}{=} \quad \frac{4}{b} \int_{0}^{\bar{u}} u z d u \stackrel{\mathrm{CV} 3}{=} 4 \int_{o}^{\bar{u}} v d u \\
& \stackrel{*}{=} \quad 4 \int_{0}^{\bar{v}} u d v \stackrel{\mathrm{CV}}{=} \frac{4}{b} \int_{0}^{\bar{v}} v w d v \\
& \stackrel{\mathrm{T}}{=} \quad \frac{2}{b} \int_{0}^{b} v^{2} d w \stackrel{\mathrm{CV} 5}{=} 2 \int_{0}^{b} s d w \stackrel{\mathrm{CV} 6}{=} \frac{2}{b^{k-4}} \int_{0}^{b} w^{k-4} t d w \\
& \stackrel{\mathrm{T}}{=} \frac{2}{(k-3) b^{k-4}} \int_{0}^{b} w^{k-3} d t \\
& \begin{array}{lll}
\text { CV1: } & x=z^{2} / b & \mathrm{C}_{1}: y^{2} z^{2 k}=b^{2 k}\left(z^{2}-b^{2}\right) \\
\text { CV2: } & y=u^{2} / z & \mathrm{C}_{2}: u^{4} z^{2 k-2}=b^{2 k}\left(z^{2}-b^{2}\right) \\
\text { CV3: } & z=b v / u & \left.\mathrm{C}_{3}: v^{2 k-2}=b^{4}\right)\left(v^{2}-u^{2}\right) u^{2 k-8} \\
\text { CV4: } & u=v w / b & \mathrm{C}_{4}: b^{2 k-10} v^{4}=\left(b^{2}-w^{2}\right) w^{2 k-8} \\
\text { CV5: } & v^{2}=b s & \mathrm{C}_{5}: b^{2 k-8} s^{2}=\left(b^{2}-w^{2}\right) w^{2 k-8} \\
\text { CV6: } & s=w^{k-4} t / b^{k-4} & \mathrm{C}_{6}: w^{2}=b^{2}-t^{2} .
\end{array}
\end{aligned}
$$

A few remarks are in order. First, notice that the quadrature of the initial curve ends by depending directly on the sum of the powers of the ordinates of the circle, already studied by FERMAT. Second, we see that in order to be able to apply FERMAT's method, it is necessary that $k>3$. This is the condition which, from a modern point of view, makes the improper integral

$$
\int_{0}^{\infty} \frac{b^{k+1}(x-b)}{x^{k}} d x
$$

convergent. Third, assuming that FERMAT essayed different curves of this nature, he did not choose $k=4$ for then the quadrature would have taken only five changes of variable. He also skipped the case $k=5$ for then the quadrature reduces to that of a higher parabola. Instead he chose $k=6$ which allowed him to exhibit eight changes of variablethe last three, though, are only needed for summing the third power of the ordinates of a circle. Fourth, in this example, he used for the first time a quite obvious result which can be seen as the General Theorem for the case $n=1$. The area of a figure is the same whether the sum of the ordinates is taken on the base or the sum of the abscissas is taken on the diameter. That is to say,

$$
\int x d y=\int y d x
$$

It is the step marked above with the symbol $\stackrel{*}{=}$. 
Last, to emphasize the great internal coherence of the Treatise, it is worth noting that this final example is the quadrature of a curve of the same class as the first he had used to obtain the quadrature of the folium. So, this last example closes the paper with a spectacular display of his method and, at the same time closes a circle returning to the starting point.

His last words clearly show the pride of the author for his creation:

We have thus used up to nine different curves to reach the knowledge of the first.

\section{Conclusions}

One of the more momentous conquests of the first third of the seventeenth century was the expression of a curve by the means of a mathematical equation which, excluding power series, reduced to a polynomial.

In fact, if a general method for determining properties of curves from their algebraic equations could be devised, a giant step would have been taken, since in this case, little by little, important parts of mathematics would achieve their independence from pure geometry.

In this direction, Descartes' finding in La Géométrie is crucial: as a curve can be expressed by the use of a polynomial equation, $P(x, y)=$ 0 , the normal at a given point $\left(x_{0}, y_{0}\right)$ of the curve can be found. The method consists of cutting the curve with a circle of center $O=(r, s)$ and imposing that the resulting polynomial $Q(x)=0$ had $x=x_{0}$ as a double root. A great success for a good method. It always depends, of course, on the degree of the polynomial equation of the curve.

More or less at the same time, the geometers of the seventeenth century came to realize the importance of squaring the curves of the form $y^{m}=b^{m \pm n} x^{\mp n}$. They devoted a great deal of energy to achieve these quadratures and they strived to find

$$
\int_{0}^{b} x^{n} d x, \quad \int_{b}^{\infty} x^{-n} d x, \int_{0}^{b} x^{ \pm m / n} d x .
$$

So, from Cavalieri to Newton and Leibniz, with different techniques and different epistemological frameworks, all their calculations led to

$$
\int_{0}^{b} x^{ \pm m / n} d x=\frac{b^{ \pm m / n+1}}{ \pm m / n+1},
$$

except the case in which the exponent is -1 . The success was so spectacular that NEWTON considered as the explicit analytical expression of a function its power series expansion and thus developed a sort of algebra of infinite series (see (19, p. 107)).

It is precisely in this context where FERMAT's contributions to algebraic geometry, tangents to curves, lengths of curves and quadratures have to be analyzed. Especially this last subject, the quadrature 
of curves, as shows the Treatise we have just reread. In a first part he establishes a general method to find the quadrature of all higher parabolas and hyperbolas. Next, he sets himself the problem of determining the quadrature of an algebraic curve given by an implicit equation $P(x, y)=0$ using the known quadratures and assuming the quadrature of a circle of radius $b$ as known.

To achieve this end, he seeks a new curve, quadrable, whose quadrature is expressible through the known quadratures of the curves at his disposal, the higher parabolas and hyperbolas.

Thus, given an equation of the form

$$
y^{n}=\sum a_{i} x^{i}+\sum b_{j} / x^{j}
$$

FERMAT is able to obtain the sum of the $y^{n}$ through the squaring of the parabolas and hyperbolas of the right-hand side. He then applies the General Theorem to reduce the degree and proceeds to determine a new curve by a change of variable that either linearizes $\left(y^{n}=b^{n-1} u\right)$ or reduces even more the degree.

In order to enlarge the class of reducible quadratures, he has to add the circle to his stock of known quadratures. He then realizes that the squaring of curves like

$$
\left(b^{2}-x^{2}\right)^{n / 2}
$$

will lead to the possibility of squaring more curves. The case in which $n$ is even presents no problem as $y^{2}=b^{2}-x^{2}$, and for odd $n$ he manages to circumvent the difficulty of the radicals by a masterful use of his method applied to $y^{2 m+1}$, where $y^{2}=b^{2}-x^{2}$.

If we had to recap, in a general set ideas, we could summarize the essence of FERMAT's method (leaving apart the last example in which he deviates from the previous, though he maintains the spirit) as follows. FERMAT knows how to compute

$$
\int_{0}^{b} y^{n} d x
$$

either by squaring directly higher parabolas or hyperbolas, (14), or as the sum of the ordinates of a circle, (15). Now, by the General Theorem,

$$
n \int_{0}^{\bar{y}} x y^{n-1} d y
$$

He then effects a change of variable of the style

$$
x y^{n-1}=b^{n-q} u^{q}
$$

with a suitable $q$. In (14) or (15), $x$ can be replaced by $b^{n-q} u^{q} / y^{n-1}$ in order to obtain a new curve

$$
P(y, u)=0 .
$$


for which

$$
\int_{0}^{\bar{y}} u^{q} d y
$$

is computable in terms of (16). The process can be iterated until reaching

$$
\int_{\alpha}^{\beta} z d w
$$

which is the actual quadrature of a curve $F(w, z)=0$.

Strictly following the previous process, it seems that the "new" quadrable curve, $F(w, z)=0$, appears at the end of the process as a sort of surprise. FERMAT - and we hope our new reading of the Treatise will have made thus clear - is conscious that the process can be reversed at least for certain families of algebraic curves with a "standard" equation.

FERMAT's method of quadratures is, as has been shown, highly original and powerful, but only applicable to a certain class of algebraic curves. In fact this limitation to a certain family of curves and their algebraic character partly explains the sparse attention the method received in its time.

In our opinion, the history of mathematics consists of understanding the writings of great mathematicians, their internal coherence, the methodology that has been used, the extension of the methods deployed. All this independently of the limited success of those writings. A paradigmatic text in this sense is the Lettres de Dettonville by Blaise Pascal. Fermat's Treatise on quadratures is another one which we hope we have contributed to vindicate at least for its great intellectual value. Our new reading is neither a historiographic analysis of FERMAT's text nor a study of its ulterior influence - which has been almost non-existent, but it offers a complete detailed analysis of all of its examples showing its inter-dependence and the logical thread that conducts them all. In some occasions we dare reconstruct obscure parts of FERMAT's exposition but we do so in the hope that these reconstructions shed some light on the method FERMAT is trying to construct.

\section{REFERENCES}

1. Kirsti Andersen, Cavalieri's method of indivisibles, Arch. Hist. Exact Sci. 31 (1985), no. 4, 291-367.

2. A. Aubry, Essai sur l'histoire de la géométrie des courbes, Annaes Scientificos da Academia Polytechnica do Porto 4 (1909), no. 2, 65-112.

3. __ Méthode de Fermat pour la quadrature des courbes, 1912, in (21, "Notes mathématiques et complements", vol.4 p. 228-230. Note XXVI). 
4. Johannis Bernoulli, Lectiones mathematicae, de methodo integralium, aliisque, Marci-Michaelis Bousquet, Laussane \& Geneve, 1757, in $(5$, vol. 3$)$.

5. __ Opera Omnia, 4 vols., Georg Olms, Hildesheim, 1968, editor, J. E. Hofman.

6. Carl B. Boyer, Fermat's integration of $X^{n}$, Nat. Math. Mag. 20 (1945), 29-32.

7. J. A. Bullard, Problem 410, Amer. Math. Monthly 23 (1916), no. 6, 210.

8. J. M. C. Duhamel, Mémoire sur la méthode de maxima et minima de Fermat et sur les méthodes des tangentes de Fermat et Descartes, Mémoires de l'Académie des Sciences de l'Institut Impérial de France 32 (1864), 269-330.

9. P. de Fermat, De cissoide fragmentum, 1662, in (21, vol. 3, pp. 285-288).

10. _ Varia opera mathematica D. Petri de Fermat Senatoris Tolosani. Acceserunt selectae quaedam ejusdem Epistolae, vel ad ipsum à plerisque doctissimis viris Gallicè, Latinè, vel Italicè, de rebus ad Mathematicas disciplinas aut Physicam pertinentibus scriptae., Joannis Pech, Toulouse, 1679, repr. Brussels, 1969.

11. __ De aequationum localium transmutatione et emendatione ad multimodaum curvilineorum inter se vel cum rectilineis comparationem, cui annectitur proportionis geometricae in quadrandis infinitis parabolis et hyperbolis usus, c. 1659, in (21, vol. 3, pp. 216-237).

12. Paolo Freguglia, On the principle of dimensional homogeneity between the 16th and the 17th century, Boll. Unione Mat. Ital. Sez. B Artic. Ric. Mat. (8) 2 (1999), no. 1, 143-160.

13. Enrico Giusti, Bonaventura Cavalieri and the theory of the indivisibles, Cremonese, Bologna, 1980, Preface to the facsimile of Exercitationes geometricae sex by Bonaventura Cavalieri.

14. S. I. B. Gray and Tagui Malakyan, The Witch of Agnesi. A lasting contribution from the first surviving mathematical work written by a woman: a commemorative on the 200th anniversary of her death, College Math. J. 30 (1999), no. 4, 258-268.

15. M. S. Mahoney, The Mathematical Career of Pierre de Fermat, 1601-1665, 2nd ed., Princeton University Press, Princeton, 1994, 1st ed. 1973.

16. T. F. Mulcrone, The names of the curve of Agnesi, Amer. Math. Monthly 64 (1957), 359-361. MR 18,982a

17. J. Paradis, J. Pla, and P. Viader, Fermat and the quadrature of the folium of Descartes, Amer. Math. Monthly 111 (2004), no. 3, 216-229.

18. Blaise. Pascal, Oeuvres complètes. L'intégral, Éditions du seuil, Paris, 1963. 
19. John Stillwell, Mathematics and its history, Undergraduate Texts in Mathematics, Springer-Verlag, New York, 1989.

20. D. J. Struik (ed.), A source book in mathematics, 1200-1800, Princeton Paperbacks, Princeton University Press, Princeton, NJ, 1986, Reprint of the 1969 edition.

21. Paul Tannery and Charles Henry (eds.), Euvres de Pierre Fermat, 4 vols. plus suppl., Gauthier-Villars, Paris, 1894-1912.

22. C. Truesdell, Maria Gaetana Agnesi, Arch. Hist. Exact Sci. 40 (1989), no. 2, 113-142.

23. F. Viète, In artem analyticem isagoge, Elzevier Press, Leyden, 1646.

24. H. G. Zeuthen, Notes sur l'histoire des matémathiques, (suite) IV: Sur les quadratures avant le calcul intégral, et en particulier sur celles de Fermat, Oversigt over det Kongelige Danske Videnskabernes Selskabs. Forhandlinger (1895), 37-80, part 4 of a paper started in 1893 in the same Bulletin.

Jaume Paradís. E-mail: jaume.paradis@upf.edu;

Pelegrí Viader. E-mail: pelegri.viader@upf.edu;

Dept. d'Economia i Empresa, Universitat Pompeu Fabra, Barcelona, SPAIN.

Josep Pla. E-mail: pla@cerber.mat.ub.es Dept. de Lògica i Història de la Ciència, Universitat de Barcelona, Barcelona, SPAIN 